\title{
Building of a composite virtual slide from contiguous tissue samples
}

\author{
Benoît Plancoulaine ${ }^{1,3 \dagger}$, Myriam Oger ${ }^{1,2^{*} \dagger}$, Nicolas Elie ${ }^{1,3}$, Philippe Belhomme ${ }^{1,3}$, Paulette Herlin ${ }^{1,2}$, Abir Nasri $^{2}$, \\ Célia Augé ${ }^{1,2}$, Mylène Brécin ${ }^{2}$, Jacques Marnay ${ }^{1,2}$, Catherine Bor-Angelier ${ }^{1,2}$ \\ From 12th European Congress on Digital Pathology \\ Paris, France. 18-21 June 2014
}

\begin{abstract}
Background: Currently available microscope slide scanners produce whole slide images at various resolutions from histological sections. Nevertheless, acquisition area and so visualization of large tissue samples are limited by the standardized size of glass slides, used daily in pathology departments. The proposed solution has been developed to build composite virtual slides from images of large tumor fragments.

Materials and methods: Images of HES or immunostained histological sections of carefully labeled fragments from a representative slice of breast carcinoma were acquired with a digital slide scanner at a magnification of 20x. The tiling program involves three steps: the straightening of tissue fragment images using polynomial interpolation method, and the building and assembling of strips of contiguous tissue sample whole slide images in $x$ and $y$ directions. The final image is saved in a pyramidal BigTiff file format. The program has been tested on several tumor slices. A correlation quality control has been done on five images artificially cut.
\end{abstract}

Results: Sixty tumor slices from twenty surgical specimens, cut into two to twenty six pieces, were reconstructed. A median of $98.71 \%$ is obtained by computing the correlation coefficients between native and reconstructed images for quality control.

Conclusions: The proposed method is efficient and able to adapt itself to daily work conditions of classical pathology laboratories.

\section{Background}

Microscopes associated with modern acquisition systems, such as video camera, give the pa-thologist opportunity to work, on screen, at different magnifications [1]; however it is still difficult to visualize, in one time, the entire histological preparation. Since less than two decades [2], microscope slide scanner devices give access to an image of the whole histological section [3], so-called "Whole Slide Image" (WSI). It allows a quick inspection of the tissue fragment by panning into its entire section surface and by zooming progressively across the continuous magnification scale [4], thanks to a virtual slide

\footnotetext{
* Correspondence: m.oger@baclesse.unicancer.fr

† Contributed equally

'Normandie Université; UNICAEN, CLCC F. Baclesse, PATHIMAGE BioTICLA EA 4656, Caen, France

Full list of author information is available at the end of the article
}

viewer. Nevertheless, despite this technological advance, the tissue surface that can be observed on the computer screen is classically limited to the dimensions of the standard glass slides and glass coverslips used daily in pathology laboratories. It is always possible to generate a WSI of large tissue fragments, using large slides and microscope slide scanners that accept them. However, the technical and economic constraints limit the use of this technology to research studies and specialized laboratories and can be hardly introduced in a routine pathology department. Recently published papers described a technique to rebuild a whole prostatic tumor section from WSI of four contiguous tissue fragments of standard size $[5,6]$, using a mainly interactive method.

The aim of the present paper is to detail a solution able to give one high resolution large image of the whole histological section of big lesions, called Composite Virtual 
Slide (CVS), rebuilt from any number of WSI of contiguous tissue fragments. A quality control protocol was settled in addition, in order to evaluate the quality of the results more precisely than only visually.

\section{Materials and methods}

\section{Tissue samples}

The solution was elaborated from surgical specimens of invasive breast carcinoma, 20 primary tumors and 1 metastatic lymph node, collected since 2011, from patients followed at the François Baclesse Cancer Centre.

\section{Sample preparation}

The most representative slice of each surgical specimen is selected during the macroscopic examination. The whole slice is cut into several pieces according to a regular grid of rectangles whose size should fit those of a standard disposable embedding cassette $(31 \mathrm{~mm} \times$ $25 \mathrm{~mm} \times 5 \mathrm{~mm}$ ). At this stage, each fragment is oriented and carefully labeled according to its localization recorded on a photographic map. After paraffin embedding, each fragment is trimmed into 3 to $5 \mu \mathrm{m}$ thick section; each thin paraffin section is spread over the glass slide, while carefully avoiding its upturn, and then submitted to standard protocols.

\section{Staining}

The histological sections were stained according to the Hematoxylin-Erythrosine-Saffron (HES), Periodic Acid Schiff (PAS) and/or immunostained for hormonal receptors (ER and PR), HER2, proliferation markers (Ki-67 and $\mathrm{PHH} 3$ ), vascular marker (CD31), using automatons and standard protocols of the pathology department.

\section{Image acquisition}

Whole slide images of histological sections were digitized at $20 \times$ using the ScanScope $\mathrm{CS}^{\circledR}$ microscope slide scanner from Aperio Technologies. They were recorded as tiled tiff images.

\section{Image processing for WSI-tiling}

Taking into account the large size of the WSI, a two resolution procedure has been developed. A first composite image is built at a low resolution. The user is allowed, at this step, to correct the automatic stitching of the fragments if needed. Then, the final composite virtual slide is assembled at full resolution by reference to the parameters of the previous adjustment done at low resolution.

First, the program extracts a low resolution sub-image ( $8 \mu \mathrm{m}$ per pixel) from the WSI pyramid of each fragment; it corresponds to a sixteen fold ( $\mathrm{x}$ and $\mathrm{y}$ ) down-sampling of the full resolution image $(0.5 \mu \mathrm{m}$ per pixel). Then it constructs the corresponding untiled binary mask of the tissue and computes its edges. The fully automatic program builds thereafter horizontal strips by bringing together recursively binary images of tissue fragments from the left to the right according to the original map. For each strip, it straightens first the fragment on the left to align its right-side edge pixels; it then straightens the fragment on the right to align its left-side edge pixels. The two fragments are thereafter registered by matching the right-side edge center of the first straightened fragment with the left-side edge center of the second straightened fragment of the tissue section (Figure 1a). Finally, the low resolution composite binary mask is built by assembling recursively downwards the horizontal strips of fragments previously stitched: the application straightens the top strip to align its bottom-part pixels, straightens the bottom strip to align its top-part pixels, then registers both strips by matching bottom-side center of the straightened top strip with the up-side center of the straightened bottom strip (Figure 1b).

A low resolution untiled color image is built in parallel taking the straightened binary masks as a reference, in order to visually evaluate the reconstruction result. At this step, if needed, the user can correct the original binary masks as well as the stitched strips with an image editing software in order to reduce artifacts at the edges. Since the algorithm uses the binary masks including any adjustments, the user can repeat this low resolution correction until a satisfactory reconstruction is obtained.

For the construction of the color high resolution composite virtual slide, each WSI and each strip are straightened and stitched by reference to the distortion parameters of the low resolution binary masks, refined by a polynomial interpolation. The final CVS is provided as a tiled "BigTiff" image.

The program was developed in $C$ language without any parallelization and ran on 32 and 64 bits personal computers under Windows and Linux operating systems.

\section{Quality control protocol}

This protocol consists of four steps which were applied on five breast tumors to evaluate the internal deformation of tissue structures. These tumors were chosen in order to get a representative whole section on a single glass slide (HES staining). First, the WSI was split in several pieces ( 4 to 17). Then, the entire section was reconstructed thanks to WSI-tiling program. Finally, each obtained CVS was compared to the original WSI. Due to the huge size of the images the comparison was done on a representative sample of them. For this purpose, a random systematic sampling (spacing of 2500 pixels) was done on the paired images, WSI and corresponding CVS. This sampling allows the extraction of a high resolution gallery of representative thumbnails of 


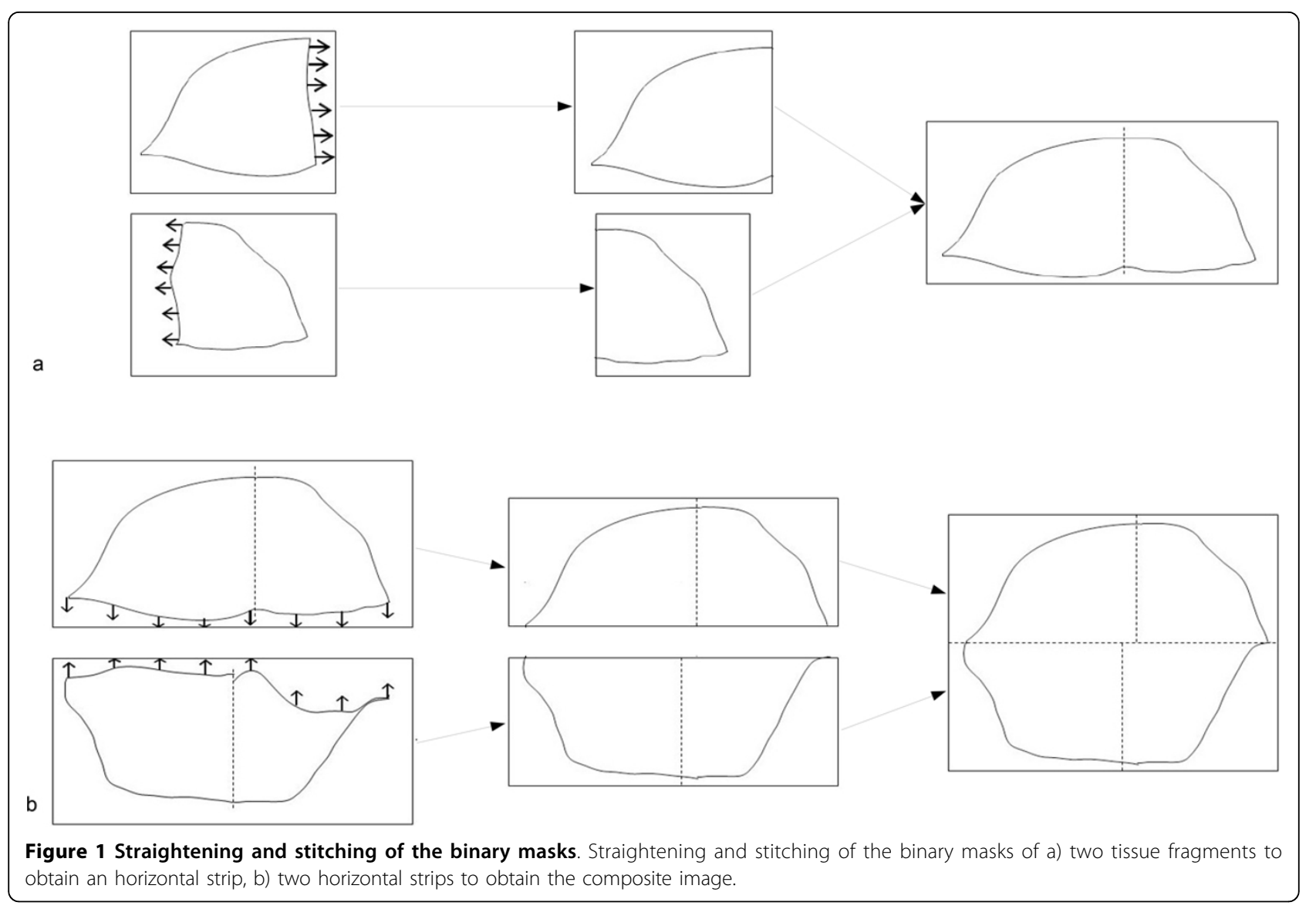

$500 \times 500$ pixels for each image. Each pair of thumbnails was registered [7] and the correlation coefficient was computed between these registered thumbnails. This procedure was reiterated four times.

Another control was done on images with less than 6 pieces to evaluate the influence of dis-tortions by rotation and homothety of each piece.

\section{Results}

The full strategy used for getting Composite Virtual Slides is illustrated Figure 2.

Sixty images of a whole histological section of twenty slices of breast tumors were built from 2 to 26 WSI (table 1). Few seconds for 2 WSI, to five minutes for 26 WSI, were necessary to obtain a low resolution untiled color image. The automatic high resolution procedure, working on only one processor, has taken 6 hours for a CVS built from 10 WSI (patient \#2, table 1) using a PC running under 64 bits operating system, with $2.4 \mathrm{GHz}$ of i7 CPU and $16 \mathrm{~Gb}$ of RAM.

Figure 3 illustrates the visual quality of the straightening of boundaries and internal tissue of WSI inside the final CVS. During the stitching process, when the limits of the tissue fragments are uneven, some small parts of the limits of the tissue can be lost.
Results of the quality control are presented Figure 4 and table 2. Figure 4 illustrates, for one of the tested cases and one random systematic sampling, the correlation obtained, thumbnail per thumbnail, between galleries of the original image and the corresponding CVS. In this example, the majority of thumbnails exhibits a correlation coefficient close to $100 \%$. The worse correlation coefficients are obtained from background white thumbnails, for which no registration could be done. The table 2 gives the median of the correlation coefficients of thumbnails coming from the CVS rebuilt from non-distorted WSI. The median of the correlation coefficients of the five cases for non-distorted WSI is $98.71 \%$. After rotation or homothety this median falls respectively to $87.57 \%(\mathrm{n}=2)$ and $85.90 \%(\mathrm{n}=2)$ and reaches $80.73 \%$ when combining the two $(n=3)$.

\section{Discussion}

The proposed solution has proved its efficacy to rebuild composite high resolution virtual slides of very large breast tumor slices cut into several tissue fragments included in classical embedding cassettes.

The reconstruction of the high resolution CVS is fully automatic, but at low resolution, a user intervention is possible to improve the stitching, if necessary. The WSI- 

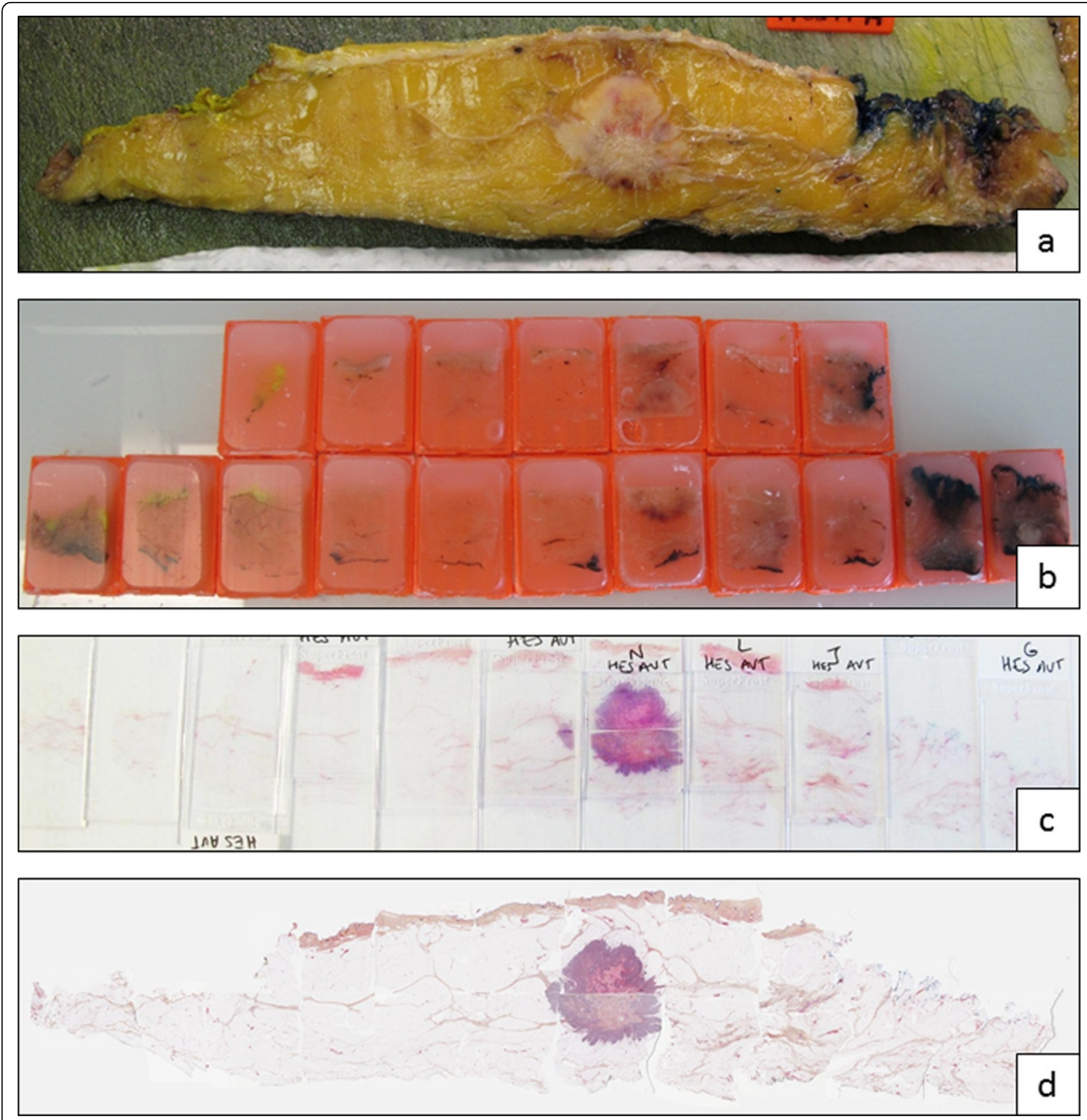

Figure 2 From surgical specimen to Composite Virtual Slide. a) Most representative slice of the surgical specimen; b) embedding cassettes containing all pieces of the entire slice; c) $5 \mu \mathrm{m}$ histological sections stained according to HES protocol; d) Composite Virtual Slide obtained after using the WSI-tiling strategy.

Table 1 Details of the 60 reconstructed images, ranked according to the size of the tissue slice.

\begin{tabular}{|c|c|c|c|c|c|c|}
\hline & \# Patient & Staining & \# of WSI & Size in pixels & Size in GB & Size of the slice $(\mathrm{mm})$ \\
\hline 1 & 1 & HES & 25 & $246576 \times 425856$ & 293.4 & $230 \times 110$ \\
\hline 2 & & HES & 26 & $246576 \times 556080$ & 383.1 & \\
\hline 3 & 2 & Ki67 & 10 & $101040 \times 373024$ & 105.3 & $200 \times 55$ \\
\hline 4 & 3 & HES & 18 & $104384 \times 513664$ & 149.8 & $200 \times 40$ \\
\hline 5 & & HES & 6 & $104400 \times 142592$ & 41.6 & \\
\hline
\end{tabular}


Table 1 Details of the 60 reconstructed images, ranked according to the size of the tissue slice. (Continued)

\begin{tabular}{|c|c|c|c|c|c|c|}
\hline 6 & & ER & 6 & $104048 \times 155248$ & 45.1 & \\
\hline 7 & & PR & 6 & $104400 \times 143392$ & 41.8 & \\
\hline 8 & & Ki67 & 6 & $98992 \times 139424$ & 38.6 & \\
\hline 9 & & HES & 6 & $104400 \times 142592$ & 41.6 & \\
\hline 10 & 4 & HES & 8 & $250272 \times 42816$ & 29.94 & $200 \times 30$ \\
\hline 11 & & HES & 8 & $285376 \times 83792$ & 66.8 & \\
\hline 12 & 5 & HES & 14 & $99680 \times 449120$ & 125.1 & $170 \times 40$ \\
\hline 13 & 6 & HES & 17 & $332976 \times 144848$ & 134.8 & $150 \times 80$ \\
\hline 14 & 7 & HES & 11 & $145792 \times 286464$ & 116.7 & $140 \times 70$ \\
\hline 15 & & ER & 11 & $143984 \times 298256$ & 120 & \\
\hline 16 & & PR & 11 & $145792 \times 298992$ & 121.8 & \\
\hline 17 & & Ki67 & 11 & $147584 \times 311872$ & 128.6 & \\
\hline 18 & & HER & 11 & $143984 \times 298256$ & 120 & \\
\hline 19 & & HES & 7 & $151184 \times 122064$ & 51.6 & \\
\hline 20 & & HES & 17 & $161667 \times 381488$ & 172.3 & \\
\hline 21 & 8a (Tumor) & ER & 9 & $149392 \times 193264$ & 80.7 & $85 \times 65$ \\
\hline 22 & & $P R$ & 7 & $147584 \times 145888$ & 60.2 & \\
\hline 23 & & Ki67 & 7 & $150192 \times 148928$ & 62.5 & \\
\hline 24 & 8b (Metastasis) & HES & 4 & $109792 \times 96384$ & 29.6 & $45 \times 50$ \\
\hline 25 & & PR & 4 & $109792 \times 93248$ & 28.6 & \\
\hline 26 & 9 & HES & 6 & $194384 \times 88800$ & 48.2 & $82 \times 35$ \\
\hline 27 & 10 & HES & 3 & $163792 \times 51280$ & 23.5 & $80 \times 25$ \\
\hline 28 & 11 & HES & 3 & $62432 \times 141296$ & 24.6 & $70 \times 30$ \\
\hline 29 & 12 & HES & 3 & $134384 \times 57680$ & 21.7 & $65 \times 35$ \\
\hline 30 & & ER & 3 & $58896 \times 122928$ & 20.2 & \\
\hline 31 & & $P R$ & 3 & $55456 \times 128640$ & 19.9 & \\
\hline 32 & & Ki67 & 3 & $63600 \times 127392$ & 22.6 & \\
\hline 33 & & HER & 3 & $64528 \times 122288$ & 22 & \\
\hline 34 & & HES & 3 & $56256 \times 135200$ & 21.3 & \\
\hline 35 & 13 & HES & 4 & $115200 \times 82997$ & 26.7 & $60 \times 40$ \\
\hline 36 & & HES & 4 & $116752 \times 88896$ & 29 & \\
\hline 37 & & ER & 4 & $109800 \times 90343$ & 27.7 & \\
\hline 38 & & PR & 4 & $109800 \times 91497$ & 28.1 & \\
\hline 39 & & Ki67 & 4 & $115200 \times 94360$ & 30.4 & \\
\hline 40 & & PAS & 4 & $117000 \times 86126$ & 28.2 & \\
\hline 41 & & $\mathrm{PHH} 3$ & 4 & $120600 \times 90403$ & 30.5 & \\
\hline 42 & & CD31 & 4 & $117000 \times 93036$ & 30.4 & \\
\hline 43 & 14 & HES & 4 & $118784 \times 90032$ & 29.9 & $55 \times 35$ \\
\hline 44 & & ER & 4 & $122400 \times 88240$ & 30.2 & \\
\hline 45 & & PR & 4 & $122384 \times 89216$ & 30.5 & \\
\hline 46 & & Ki67 & 4 & $116992 \times 88928$ & 29.1 & \\
\hline 47 & 15 & HES & 2 & $100800 \times 48016$ & 13.5 & $55 \times 25$ \\
\hline 48 & & $E R$ & 2 & $98992 \times 49584$ & 13.7 & \\
\hline 49 & & $P R$ & 2 & $95392 \times 49744$ & 13.3 & \\
\hline 50 & & HES & 2 & $100784 \times 48336$ & 13.6 & \\
\hline 51 & 16 & HES & 2 & $72000 \times 82544$ & 16.6 & $50 \times 35$ \\
\hline 52 & & ER & 2 & $61120 \times 79440$ & 13.6 & \\
\hline
\end{tabular}


Table 1 Details of the 60 reconstructed images, ranked according to the size of the tissue slice. (Continued)

\begin{tabular}{|c|c|c|c|c|c|c|}
\hline 53 & & PR & 2 & $61200 \times 81744$ & 14 & \\
\hline 54 & & Ki67 & 2 & $62992 \times 80336$ & 14.1 & \\
\hline 55 & & HES & 2 & $73184 \times 85488$ & 17.5 & \\
\hline 56 & 17 & HES & 2 & $52928 \times 89344$ & 13.2 & $50 \times 25$ \\
\hline 57 & & HES & 2 & $52192 \times 87456$ & 12.8 & \\
\hline 58 & 18 & HES & 2 & $91792 \times 40688$ & 10.4 & $50 \times 20$ \\
\hline 59 & 19 & HES & 2 & $59808 \times 88256$ & 14.7 & $45 \times 25$ \\
\hline 60 & 20 & HES & 2 & $66816 \times 83872$ & 15.7 & $40 \times 30$ \\
\hline
\end{tabular}

tiling procedure is ready for use and accepts classical Tiff as well "BigTiff" tiled images.

The high resolution reconstruction is long, but the decrease of the process time is to be con-sidered by optimizing the code with the introduction of parallelization in order to take advantage of the multi-cores available on classical PC.

The chosen quality control strategy showed satisfactory quantitative performances. Even if the performances of the proposed solution lightly decrease when the fragments are previously distorted, the correlation coefficient is still high, and the quality of the final image is almost identical to the initial image. This decrease can come from both the artificial distortion (interpolation effect) and the straightening used by the here proposed solution.

To limit the distortions inside the final CVS, coming from initial deformations of tissue fragments, it is then mandatory to carefully orientate tissue fragments in the embedding cassettes as well as paraffin ribbons on glass slides.

Even if some scanner devices can accept large slides, the benefit of the proposed solution is its ability to adapt to the daily work conditions and economic constraints of common pathology departments.

To the best of our knowledge, two papers of the same team have already described a tech-nique to rebuild the whole prostatic tumor section from WSI of contiguous

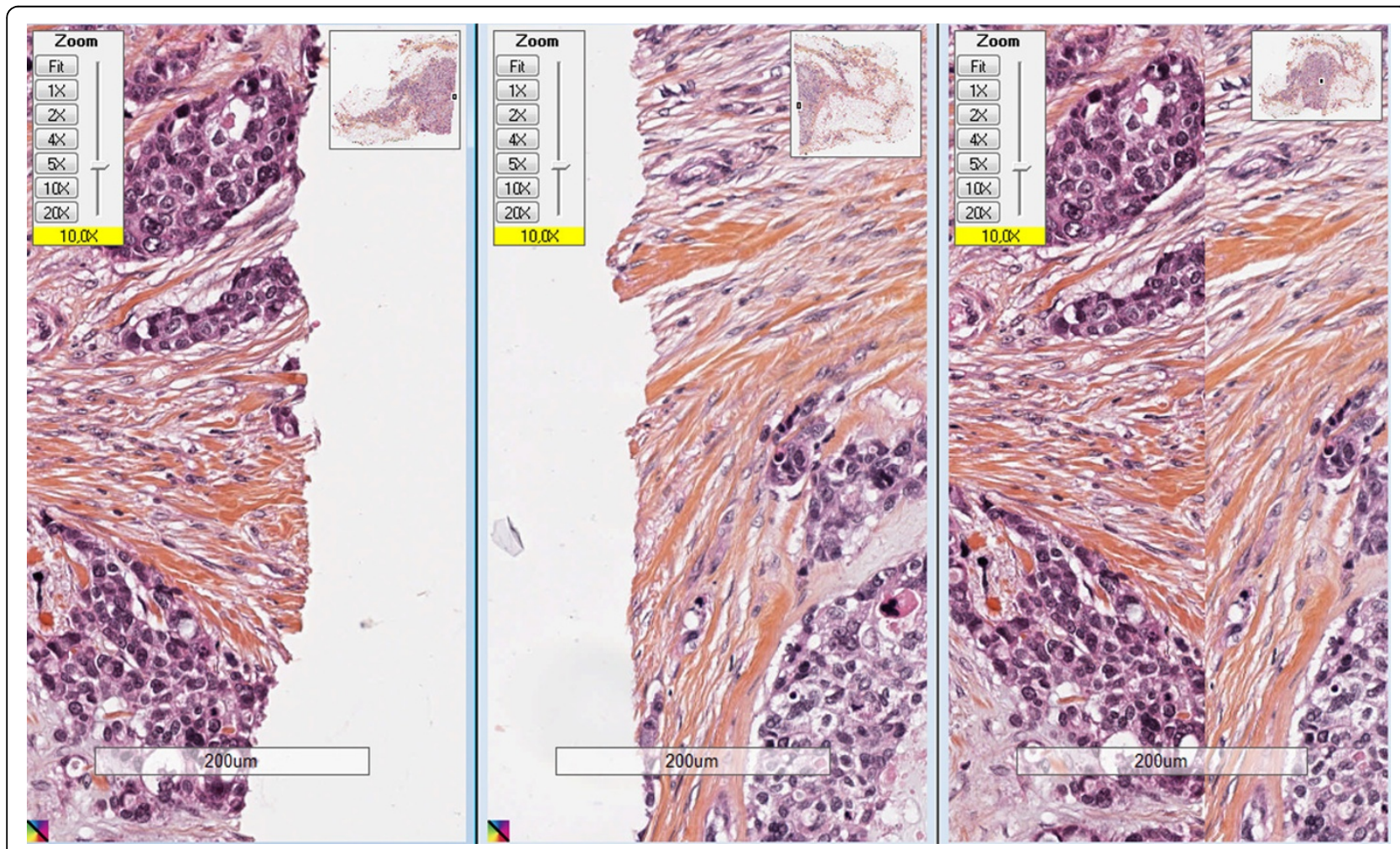

Figure 3 Detail of a stitching zone after WSI-tiling strategy. Left: detail of the left WSI; center: detail of the right WSI; right: final CVS coming from two WSI. 


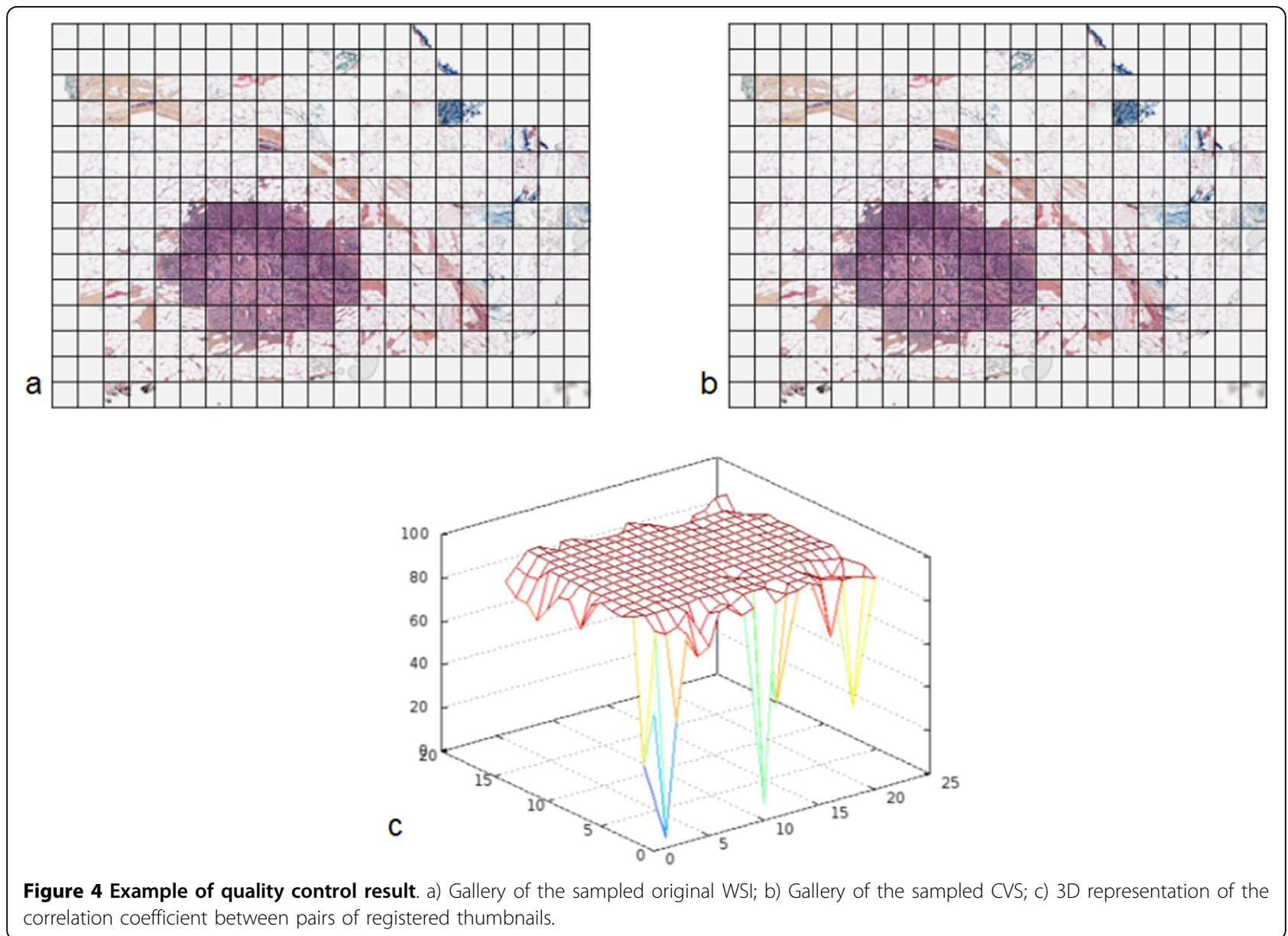

Table 2 Quality control results: Correlation coefficients computed on pairs of high resolution thumbnail galleries from CVS

\begin{tabular}{|c|c|c|c|c|}
\hline Image & number of fragments per image & Sampling & number of thumbnails per gallery & Correlation coefficient \\
\hline \multirow[t]{4}{*}{ Image 1} & 5 & $1^{\text {st }}$ & 90 & $98.70 \%$ \\
\hline & & $2^{\text {nd }}$ & 90 & $98.51 \%$ \\
\hline & & $3^{\text {rd }}$ & 90 & $98.45 \%$ \\
\hline & & $4^{\text {th }}$ & 90 & $98.44 \%$ \\
\hline \multirow[t]{4}{*}{ Image 2} & 4 & $1^{\text {st }}$ & 20 & $98.14 \%$ \\
\hline & & $2^{\text {nd }}$ & 16 & $97.26 \%$ \\
\hline & & $3^{\text {rd }}$ & 16 & $97.40 \%$ \\
\hline & & $4^{\text {th }}$ & 16 & $98.46 \%$ \\
\hline \multirow[t]{4}{*}{ Image 3} & 17 & $1^{\text {st }}$ & 315 & $99.23 \%$ \\
\hline & & $2^{\text {nd }}$ & 330 & $99.25 \%$ \\
\hline & & $3^{\text {rd }}$ & 315 & $99.23 \%$ \\
\hline & & $4^{\text {th }}$ & 315 & $99.22 \%$ \\
\hline \multirow[t]{4}{*}{ Image 4} & 12 & $1^{\text {st }}$ & 396 & $98.70 \%$ \\
\hline & & $2^{\text {nd }}$ & 418 & $98.64 \%$ \\
\hline & & $3^{\text {rd }}$ & 396 & $98.72 \%$ \\
\hline & & $4^{\text {th }}$ & 396 & $98.84 \%$ \\
\hline
\end{tabular}


Table 2 Quality control results: Correlation coefficients computed on pairs of high resolution thumbnail galleries from CVS (Continued)

\begin{tabular}{|c|c|c|c|c|}
\hline \multirow[t]{4}{*}{ Image 5} & 6 & $1^{\mathrm{st}}$ & 238 & $99.25 \%$ \\
\hline & & $2^{\text {nd }}$ & 208 & $99.24 \%$ \\
\hline & & $3^{\text {rd }}$ & 221 & $99.27 \%$ \\
\hline & & $4^{\text {th }}$ & 221 & $99.23 \%$ \\
\hline
\end{tabular}

tissue fragments $[5,6]$. But, the proposed method is interactive, its use is limited to four fragments and up to now, it did not give access to the reconstruction of an image at a magnification higher than $10 x$. In this context, the here proposed solution has been developed to give the pathologist the ability to observe and make measurements, up to the resolution of $0.5 \mu \mathrm{m}$ (i.e. magnification of 20x), on one large image of the whole histological section of large lesions, rebuilt from any number of virtual slides of contiguous tissue fragments, while limiting at most the user inter-vention.

Specific viewer allows the "stitching" of contiguous slides without rebuilding the CVS. Nev-ertheless, to process and analyze the whole tumor slice, a rebuild CVS is necessary.

The added values of this solution are numerous for the pathologist, contributing both in rou-tine diagnosis and in research.

This solution would contribute to make an easier histological diagnosis on surgical specimen. The reconstruction of an entire section of an organ on virtual slides could help pathologist to finely evaluate the surgical margins, the invasion and growth of the lesion as well as the abnormalities of the adjacent structures morphology [8], on a faster and easier way. So this solution might potentiate the interest of using digital slides routinely.

In research, this approach could be of great interest both for correlative research with other image modalities $[8,9]$ (macro-images of the tumor section, scanner, MRI or ultra-sound images) and to better understand these images in translational research. Image analysis could help studying architectural features and heterogeneity of distribution of immunohistochemical markers on large tumors, and select cell immunophenotypic clones for genetic analysis. The registration of serial histological sections and study of co-localization of markers could be possible. So this solution might contribute to the understanding of different ways of tumor growth.

\section{Conclusions}

This solution will have further developments in order to create an interface for users and to improve speed for daily practice. As it could of course contribute to research giving topographic information on tissues, the use of such a solution is one of the first steps expected by the pathologists before adopting digital pathology for their routine diagnosis. The ability to visualize complete sections of any surgical specimen could facilitate pathologists' daily work, giving them an easy access to information, such as margins and size of tumor structures, which are time consuming steps. Even more, the use of composite virtual slide will give more accuracy to measurements; the precision of these parameters is required by oncologists for further therapeutic decisions.

\section{Competing interests}

The authors declare that they have no competing interests.

\section{Authors' contributions}

BP: Developed study conception and design, and drafted the manuscript. $\mathrm{MO}$ : Selected cases, performed data acquisition, performed image processing and quality control and drafted the manuscript. NE, PB, HP, JM: participated in the design of the study and drafted the manuscript. AN, CA, MB: Developed and performed sample preparation protocol, selected cases and performed sample preparation, slide staining and data acquisition. CB: Supervised study concept and design, selected cases, performed histopathological evaluation and drafted the manuscript. All authors read and approved the final manuscript.

\section{Acknowledgements}

The authors would like to thank any person who has contributed to this work and particularly the staff of the Pathology Department of the F. Baclesse Cancer Centre.

Research on Composite Virtual Slide Solution has been supported by the European Union (FEDER 33541). Europe is involved in Normandy with the European Regional Development Fund.

Publication of this supplement has been funded by 12th European Congress on Digital Pathology. This article has been published as part of Diagnostic Pathology Volume 9 Supplement 1, 2014: Selected articles from the 12th European Congress on Digital Pathology. The full contents of the supplement are available online at http://www.diagnosticpathology.org/ supplements/9/S1

\section{Authors' details}

${ }^{1}$ Normandie Université; UNICAEN, CLCC F. Baclesse, PATHIMAGE BioTICLA EA 4656, Caen, France. ${ }^{2}$ CLCC F. Baclesse, Pathology department, Caen, France. ${ }^{3}$ Normandie Université; UNICAEN, CMABIO-HIQ facility, Caen, France.

Published: 19 December 2014

\section{References}

1. Gabril MY, Yousef GM: Informatics for practicing anatomical pathologists: marking a new era in pathology practice. Modern Path 2010, 23:349-358.

2. Ferreira R, Moon B, Humphries J, et al: The virtual microscope. Proc AMIA Annu Fall Symp 1997, 449-53.

3. Rojo MG, Bueno García G, Peces Mateos C, González García J, Carbajo Vicente M: Critical Comparison of 31 Commercially Available Digital Slide Systems in Pathology. Int J Surg Pathol 2006, 14:285-305. 
4. Kumar RK, Velan GM, Korell SO, Kandara M, Dee FR, Wakefield D: Virtual microscopy for learning and assessment in pathology. J Pathol 2004, 204(5):613-618.

5. Toth RJ, Shih N, Tomaszewski JE, Feldman MD, Kutter O, Yu DN, Paulus JC, Paladini G, Madabhushi A: Histostitcher ${ }^{\mathrm{TM}}$ : An informatics software platform for reconstructing whole-mount prostate histology using the extensible imaging platform framework. J Pathol Inform 2014, 5:8.

6. Patel P, Chappelow J, Tomaszewski JE, et al: Spatially weighted mutual information (SWMI) for registration of digitally reconstructed ex vivo whole mount histology and in vivo prostate MRI. Conf Proc IEEE Eng Med Biol Soc 2011, 2011:6269-72.

7. Marcel B, Briot M, Murrieta R: Calcul de translation et rotation par transformation de Fourier. Traitement du signal 1997, 14(2):135-149, (in French).

8. Foschini MP, Baldovini C, Ishikawa Y, Eusebi V: The Value of Large Sections in Surgical Pathology. International Journal of Breast Cancer 2012, 2012, Article ID 785947, 7 pages.

9. Netsch T, Roesch P, van Muiseinkel A, Weese J: Toward real-time multimodality 3-D medical image registration. International Conference on Computer Vision (ICCV'01) 2001, 501-8.

doi:10.1186/1746-1596-9-S1-S9

Cite this article as: Plancoulaine et al.: Building of a composite virtual slide from contiguous tissue samples. Diagnostic Pathology 2014 9(Suppl 1):S9.

\section{Submit your next manuscript to BioMed Central and take full advantage of:}

- Convenient online submission

- Thorough peer review

- No space constraints or color figure charges

- Immediate publication on acceptance

- Inclusion in PubMed, CAS, Scopus and Google Scholar

- Research which is freely available for redistribution

Submit your manuscript at www.biomedcentral.com/submit 